\title{
Perspective of Chemical Fingerprinting of Chinese Herbs
}

Authors

Affiliations
Yi-zeng Liang ${ }^{1}$, Pei-shan $\mathrm{Xie}^{2}$, Kelvin Chan ${ }^{3}$

${ }^{1}$ Research Center of Modernization of Chinese Medicines, Central South University, Changsha, China

${ }^{2}$ Macau University of Science and Technology, Macau Institute for Applied Research in Medicine and Health, Macau, China

${ }^{3}$ CompleMED, College of Health \& Science, University of Western Sydney \& HMREC, Faculty of Pharmacy,

The University of Sydney, Sydney, Australia
Key words

- herbal medicines

- chromatographic fingerprinting

- quality control

- chemometrics

- Chinese materia medica

- TCMs

- chemical fingerprinting

\section{Abstract}

$\nabla$

The holistic system of traditional Chinese medicine (TCM) is an integrity of the ingredients contained in the Chinese herbal medicines, which creates a challenge in establishing quality control standards for raw materials and the standardization of finished herbal drugs because no single component is contributing to the total efficacy. Chromatographic fingerprinting analysis represents a rational approach for the quality assessment of TCM. It utilizes chromatographic techniques, which include CE, GC, HPLC, HPTLC, etc., to construct specific patterns for recognition of multiple compounds in TCMs. Thus, chromatographic fingerprinting analysis of herbal medicines represents a comprehensive qualitative ap-

\section{Introduction}

\section{$\nabla$}

A Western scientist, analyzing modern and Chinese medicine, reflects that "the two approaches boil down to a simple question: is it better to attack disease according to Western medicine, with a silver bullet - the one substance whose potency has been pinpointed? Or should the disease be managed by the traditional Chinese medicine (TCM) diagnosis and treatment principles using combinations of Chinese herbs to solve the problem - the holistic approach"? In fact, which one is better does depend. In general, from the point of view of science, the Western medical system, especially at theoretical level, has a much stricter and more comprehensive basis in comparison with traditional medicines which are mainly based on experiences of long history. However, a number of studies have supported the efficacy and safety of some TCM herbal formulas in a manner close to Western medicine practice [1-3]. Thus, some Western-trained scientists have experienced that the benefits of TCM drugs often come proach for the purpose of species authentication, evaluation of quality, and ensuring the consistency and stability of herbal drugs and their related products. The pragmatic comprehensive chromatographic fingerprinting analysis can disclose the detectable ingredients composition and concentration distribution under quantifiable operational conditions and therefore provide realtime quality information. It may leave a "gray" entity at the primary stage. However, consecutive study will deepen the knowledge and reduce its "gray scale", increase the transparency gradually, thereby strengthening its quality assessment potency.

as a result of synergistic interactions of multiple ingredients $[4,5]$. It follows that the reductionist approach of isolation of a single bioactive compound is not always appropriate for TCM research.

However, whatever the kind of medical system, the quality consistent medication is a prerequisite for efficacy assurance. Referring to the abovementioned, the symbolized "silver bullet" or "holistic" description is used to associate the proper assessment of the quality of Chinese herbal medicines. The conventional purpose is to identify false from true, test the impurity and determine the content of the selected single chemical entity by appropriate analytical technologies. But in recent years, the quality of judgment by determining, selecting one or two markers, ignoring the complexity of the herbal medications is no longer the trend of thought, and the consensus that the comprehensive strategy for assessing the intact quality of herbal medicine is necessary. Consequently, chromatographic fingerprinting and si- 
multaneous determination of multiple components are becoming the current trends [6-8].

\section{Chromatographic Fingerprints of Herbal Medicines $\nabla$}

Referring to the fundamental quality control of Chinese herbal medicines, the conventional reductive practice such as selecting a single chemical from the crude drugs for analyzing is unanimously recognized as an inefficient model, although the Pharmacopoeias still keep it as the mainstream quality control mode in the herbal medicine monographs. Taking Coptidis Rhizoma (Huanglian) and Phellodendron Cortex (Huangbo) as examples, both contain the major bioactive alkaloid berberine, but the efficacy of Coptis Rhizoma is mainly clearing the abnormal "hotness" incurred in the middle part of the body, while the Phellodendron bark can clear the evil "hotness" and remove "dampness" suffered in the lower part of the body according to traditional theories and practice. Berberine alone can never be responsible for the synergistic effectiveness, although such a statement appears absurd from the point of view of modern science instilled persons. In fact, it can be deduced that berberine, combined with the different various ingredients contained in the two herbals, play different roles based on their own composition. One should be aware that in TCM practice the same bioactive compounds in one plant species when combined with other TCM herbs in different TCM prescriptions produce different effects in the human body at different stages of the diseases. It is interesting to note that "individualization" in modern medical practice shares the similar concept of "varying the formulation with each individual suffering the same syndrome", as in TCM practice based on ancient theories. Therefore, getting rid of the single marker-target in assessing the herbals quality while comprehensively viewing the formula's quality by means of chromatographic fingerprint analysis (CF), can basically assure the quality of the TCMs.

In general, a representative chromatographic fingerprint generated from multiple samples of the same species is called a common pattern, which is the criterion for assessing the individual herbal material. Generally, phytoequivalence is a basic requirement for determining the efficacy.

The principles of the acceptable herbal drugs are "safety, consistency, and efficacy". When herbs of questionable origin or unreliable quality are used in all aspects of researches and treatment, it is highly unlikely that the results can be repeated and scientifically validated and will therefore be a waste of time and misleading to the investigator. Thus, chemical fingerprinting of herbal medicines, the fundamental level for their quality control, should always be taken as the top priority. Hence, the method of design for the strategy for quality assurance of TCMs should be of the most concern.

\section{Features of Chromatographic Fingerprints of Herbal Medicines $\nabla$}

In our opinion, the chromatographic fingerprint (CF) of herbal medicines has some attractive features to meet the requirements of the fundamental level for their quality control. Firstly, a CF feature is its intactness, in which all the detectable ingredients constitute an indivisible intact specific profile for identification. The intact profile strengthens the specificity of the given herbal drug, that is, not a few markers, but the total profile involving numbers of peaks, positions of peaks, and peak-to-peak ratios, which altogether contribute to a characteristic "appearance" with a group of integrated data for comparison and assessment. Secondly, CF possesses two levels of significance: the "elementary" quality control and "intensive" quality control. The former (elementary quality control) serves the routine identification and rapid overall quantifiable estimation of commercial varieties of TCM and the formulated products even if some ingredients in the fingerprint are unknown, and only if the representative profile of the authenticated sample is available; as it is unlikely we can distinguish a person from a photo without his/her personal detail information. And the latter ("intensive" quality control) refers to the ensuing in-depth studies of CF coupled with more sophisticated technologies and chemometrics and information theory (with more advanced instrument analysis, hierarchical cluster analysis, principle component analysis, and pattern recognition or $k$-nearest neighbour analysis) to enhance the high capability for processing the array of the parameters obtained from instrumental analyses. Thus, the $\mathrm{CF}$ is of great flexibility for quality control use. Finally, the CF can also serve as a "quality data bank" in which the intact groups of ingredients have been reserved for sustainable in-depth study being relevant to the traditional aspect of TCM and modern biological technologies. Thus, CF can play a QC/QA role at every developing stage of analysis. As an industrial standard or official specification of the product, its applied CF obtained from TLC, HPLC, or GC at a fundamental level can be easily accepted economically and technologically. However, some shortcomings for CF should also be mentioned, for instance, some compounds, such as polysaccharides and/or terpines, etc., cannot be detected by common HPLC technique. We believe that with the development of analytical instruments, the detection ability of chromatographies could be greatly improved for fingerprinting analysis.

\section{Examples of Case Studies by Chromatographic Fingerprinting \\ $\nabla$}

Epimedium herb (Yin yang huo in Chinese), one of the popular Chinese herbs, derives from a multiple species colony belonging to the family Berberidaceae. Most of the species are distributed in various provinces in China. There are five species of Epimedium adopted in the Chinese pharmacopoeia: E. brevicornu Maxim., E. koreanum Nakai, E. sagittatum (Sieb. et Zucc.) Maxim., E. pubescens Maxim., and E. wushanense T.S.Ying. Resources survey indicated that Epimedium pubescens, E. brevicornum, and E. koreanum are in the commercial mainstream of the CMM market. Beyond the five official species, E. acuminatum, E. leptorrhizum, and the others are also distributed in local market and could be mixed in with the first ones. Official Epimedium species have commonly C8-prenylated flavonol glycosides, which show an immunomodulatory effect, osteoblastic proliferative activity, and sex hormone activities as reported elsewhere. Therefore icariin, epimedin A, epimedin $B$, and epimedin $C$ are unanimously used as bioactive marker compounds for quality control. Hence, selecting the high-content prenylated flavonoids as specific ingredients in the Epimedium herb for identifying species and assessing the inherent quality is rational when carrying out chromatographic fingerprint analysis. The HPLC profiles of the extracts of leaves of the five official Epimedium species, namely E. koreanum, E. brevicor$n u, E$. pubescence, E. wushenense, and E. sagigattum showed the common specific region between retention times of about 37 to 

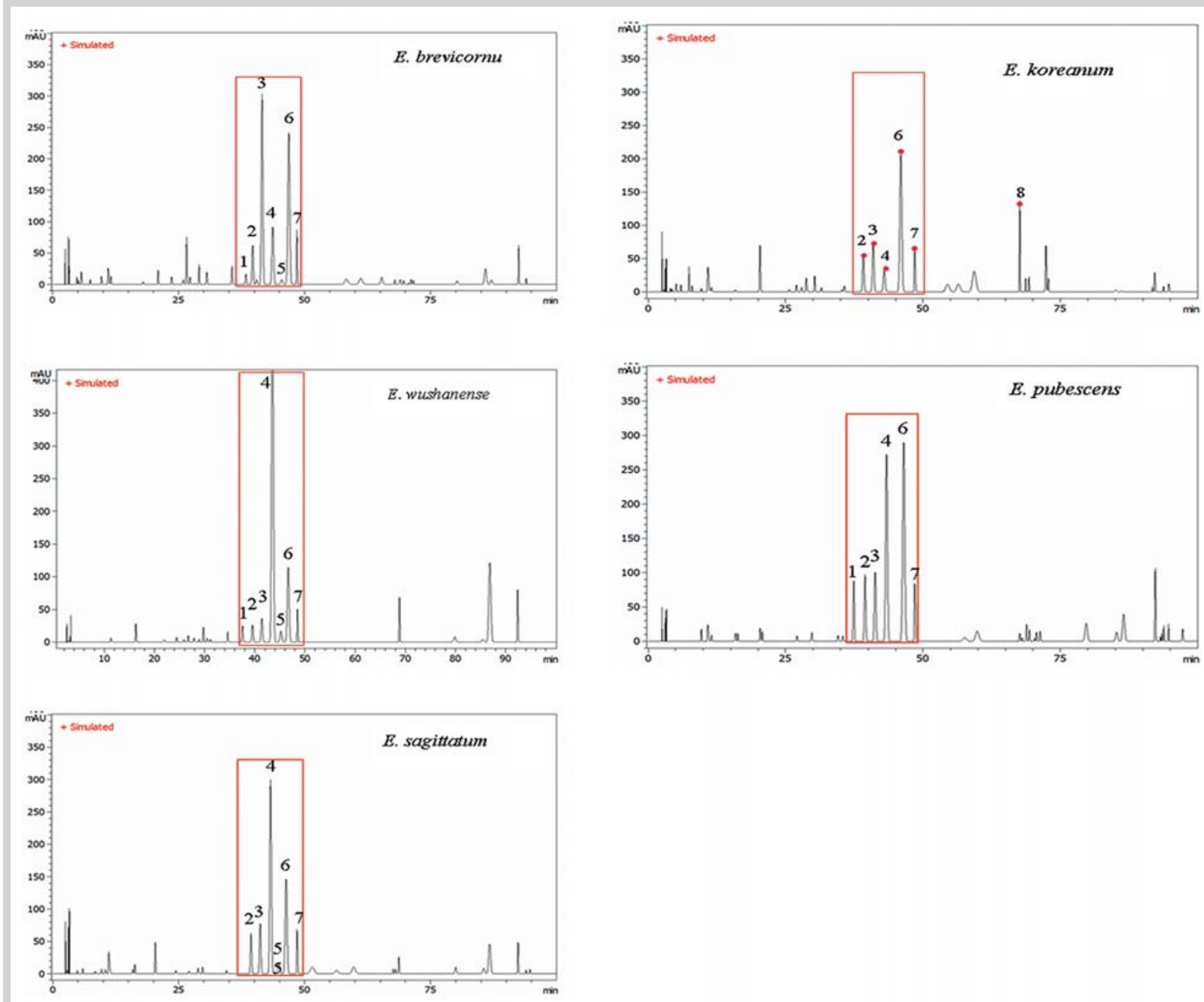

Fig. 1 HPLC profiles of the leaves of the five official Epimedium species, namely E. koreanum, E. brevicornu, E. pubescence, E. wushenense, E. sagigattum (from top to bottom). In the specific region between retention times $37 \mathrm{~min}$

- 50 min (marked by the frames), there are 5-7 peaks involving the most important four prenylated flavonoids, epimedin A (peak 2), epimedin B (peak 3 ), epimedin C (peak 4), and icariin (peak 6), respectively.
50 min, which consisted of 5 to 7 peaks involving the most important four prenylated flavonoids, i.e., epimedin A (peak 2), epimedin B (peak 3), epimedin C (peak 4), and icariin (peak 6) as well as 1 to 3 minor unknown flavonoids peaks (see 0 Fig. 1). The above-mentioned fingerprint region of HPLC profiles of Epimedium spp. represents the specificity for authentication and quality assessment. Different ratios between the peaks in this region constitute the independent characteristics of the five official species. Briefly, the dominant epimedin C peak (peak 4) in the region is characteristic of E. wushanense (E.w. pattern), icariin (peak 6) is the strongest peak found in E. koeanum (E.k. pattern) extract, epimedin B (peak 3) together with icariin (peak 6) is the attracting feature of E. brevicornum (E.b. pattern), and E. pubescens (E.p. pattern) and E. sagittatum (E.s. pattern) express their prevailing peaks of epimedin C (peak 4) and icariin (peak 6) in the region; but peak 4 is generally higher than peak 6 in the "E.s. pattern" and peak 6 is often stronger than peak 4 in the "E. p. pattern", respectively.

Using principle component analysis (PCA), analysis of 46 samples of Epimedium species, and taking the HPLC profile of E. koreanum as a reference, five patterns were categorized clearly. The first principle component $(\mathrm{PC} 1)$ is epimedin $\mathrm{C}$ (score 0.68), and the second (PC2) is epimedin B and icariin (scores 0.70 and 0.69 , respectively), this indicates that the most influential factors for Epimedium spp. is concentrated in this region. It appears that in spite of the E.b. pattern, the epimedin C (peak 4) declines from dominance in the E.w. pattern in the order of E.w. pattern $\leftarrow$ E.s. pattern $\leftarrow$ E. p. pattern $\leftarrow$ E. k. pattern (see $\bullet$ Fig. 2 for details). Previous papers published on HPLC fingerprinting analysis [9-12] of the Epimedium species focused on that region as the characteristic HPLC profile of Epimedium herbs for phytotaxonomy and quality analysis. For example, Guo and coworkers [10] attempted to delineate the subtle variation of this region of 35 species of Epimedium for differentiating the species more strictly. All the samples tested were divided into four main types and nine subtypes to compile with the complex morphological taxonomic system devised by W.T. Stearn $[13,14]$. Plant taxonomy needs a divergence strategy to distinguish the visual variance of the appearance in a subtle way to define the uniqueness of species. The complex classification verified the diversity of the botanical king- 


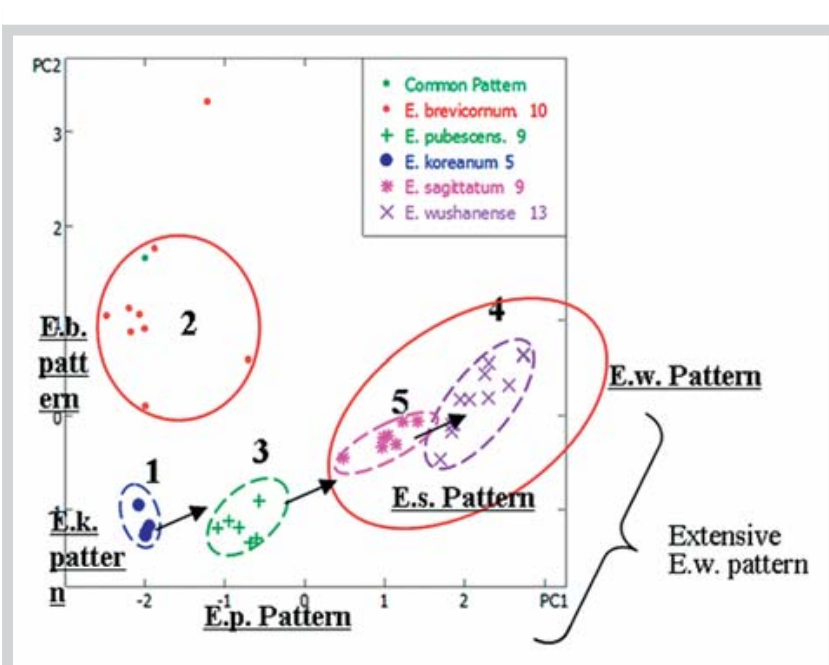

Fig. 2 The score plot of principle component analysis of 46 samples of Epimedium spp.

dom. However, for solving the practical difficulty caused by multiple species herbal medicine commodities coexisting in the CMM market, the divergence approach is obviously not the right choice. To converge the different species that possess the same HPLC fingerprint pattern towards one group will facilitate the efficacious use of multiple species of CMM such as the Epimedium species. Convergence or divergence obviously depends on the final purposes [15].

Another example for phytotaxonomy and quality analysis can be shown by the work on high-performance liquid chromatographic (HPLC) fingerprints of Fructus Aurantii immaturus (FAI) from different sources of China [16]. The fingerprints were generated in the deliberately collected 12 authentic samples and 26 commercial samples. The main bioactive compounds in this citrus herb, namely naringin and hesperidin, were first identified and quantitated. Then, principal components analysis (PCA) was performed to differentiate and classify the studied samples based on the principal peaks and entire chromatograms, respectively. Hierarchical clustering analysis (HCA) and similarity analysis (SA) were further conducted to validate the clustering results. The HPLC-DAD screening aided by chemometric analysis shows that the chemical fingerprints of 38 samples from extensive sources could be reasonably identified and systematically analyzed. This offers a new clue for the study of CMM.

To survey the distribution of the 38 samples, PCA was utilized to analyze and classify those HPLC-DAD data. The scores plot of PC1 versus PC2 was examined. First, the projection plot of PCA based on the CFs is shown in O Fig. 3. Except sample no. 30, all the others apparently form into two groups. The authentic samples (No. 13, 14, 19, and 20) from Jiangxi province and the commercial ones (No.1-4, 12, 22, 27-29) are clustered into group I, and authentic samples nos. 8, 9, 37, 38 from Hunan province, authentic ones No. 25 and 26 from Sichuan, samples 34 and 35 from Yunnan and the rest of commercial ones fall into cluster group II. All these samples studied could visibly be clustered into groups based on two sources of supply centers in Jiangxi and Hunanprovinces, respectively. CMM from different origins have different chemical properties, and thus different clusters come into being. In order to see the details in the fingerprints, the median chromatograms of the two groups of FAI are shown in 8 Fig. 4. Peaks existing in all chromatograms are considered as "common peaks", indicating the similarity among various samples. A total of 18 peaks coexisting in the 38 samples can be visually compared with the tested samples. Among the common components, the contents of hesperidin and naringin are relatively high and stable, which was confirmed with findings from the literature [17-19]. Peak $\mathrm{M}$ and $\mathrm{N}$ existing in $\odot$ Fig. $4 \mathrm{~A}$ are not found in $\triangle$ Fig. $4 \mathrm{~B}$, while peak $\mathrm{P}$ emerging in $\odot$ Fig. $4 \mathrm{~B}$ does not exist in $\odot$ Fig. 4A. The non-common peaks in each chromatogram represent the fuzziness among the same kind of CMM along with the different content of the same components existing in the samples examined. It is in accordance with the theory that the secondary metabolites of medicinal plants would clearly vary in different locations. Further work should be carried out to probe the variance of pharmacodynamic action of FAI from different origins.

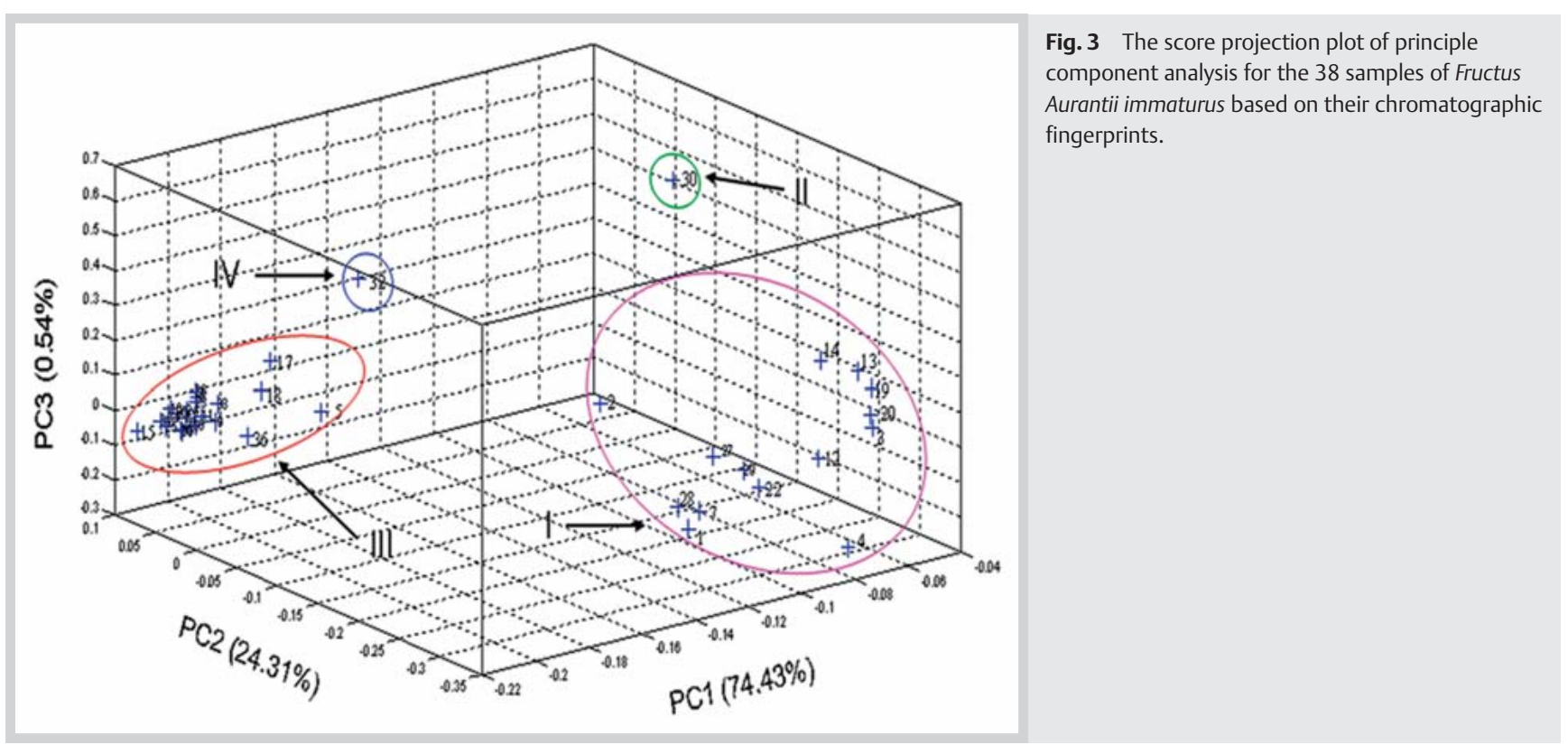




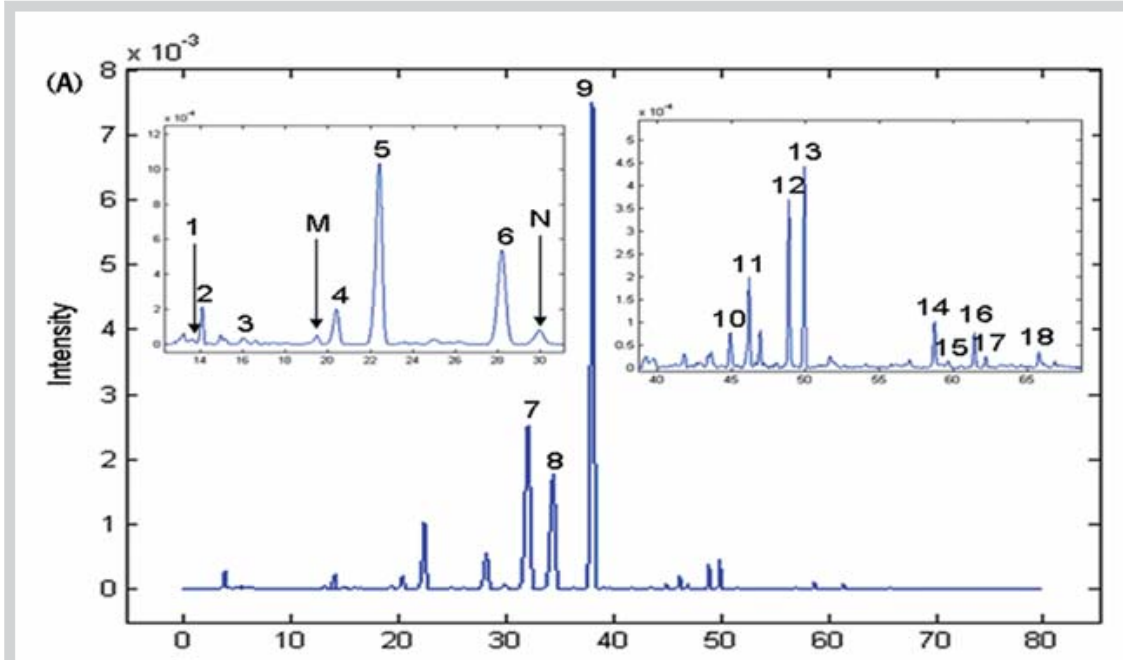

Fig. 4 Mean chromatogram of 4 authentic samples of FAl including four from jiangxi (samples nos. $12,13,19$, and 20 ) and 10 commercial samples (samples nos. 23 and 24) (A); mean chromatogram of 8 authentic samples including four from Hunan (samples nos. 08, 09, 37, and 38) and 2 from Sichuan (samples nos. 34 and 35), 2 from Yunnan (samples nos. 25 and 26), and 16 commercial samples (B).

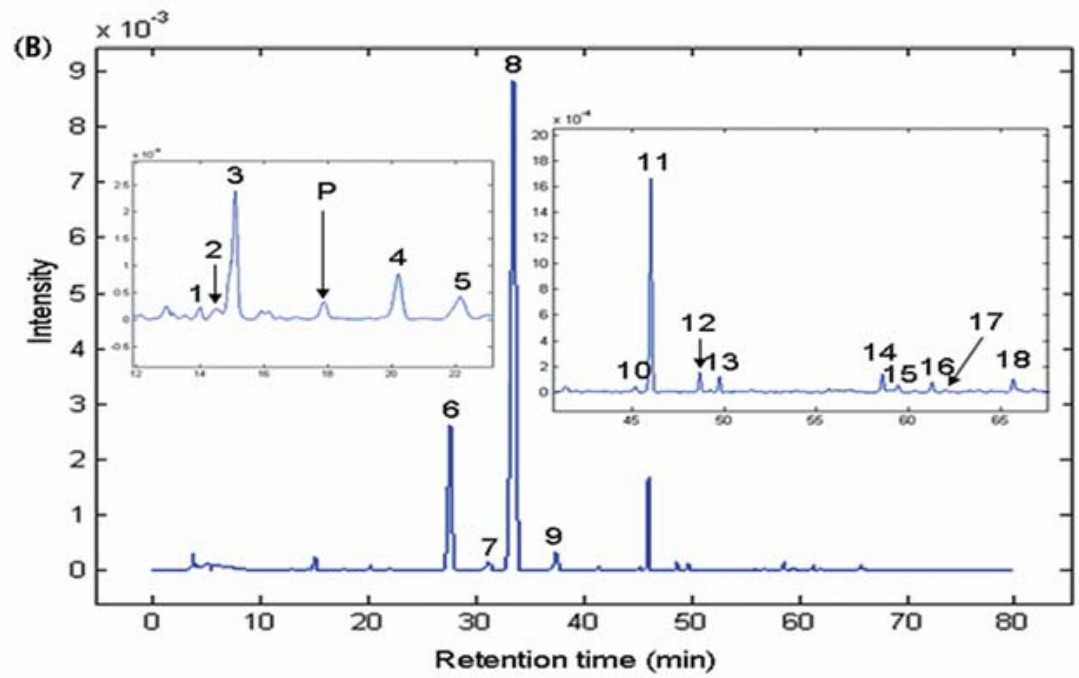

Using sulfur combustion for drying some CMM with a fleshy texture and rich starch or saccharide has for recent decades been a popular practice in some CMM processing factories for the sake of curtailing the drying process, bleaching the crude drug's darker appearance, and moth proofing. However, when the benefits are realized, potential disadvantages occur. The chromatographic fingerprint analysis of Baizhi revealed that during drying of the crude drugs by the sulfur combustion over a rather long time, the simultaneously released sulfur dioxide reacted with the ingredients of $A$. dahuricae radix (Baizhi) causing serious loss of major bioactive furocoumarins such as imperatorin, isoimperatorin, and oxypeucedanin [20].

The HPTLC fluorescence images and HPLC profiles of Baizhi with and without sulfur fumigation showed huge differences in its major ingredients. Based on the integration peak areas of the profile of sulfur-fumigated Baizhi at the major peak region, corresponding to furocoumarin and imporatorin, a loss of approximately more than $60 \%$ was detected, the most instable coumarin as oxypeucedanin disappeared along with the increase of some minor furocoumarins, which led the chromatographic fingerprint of Baizhi to be changed drastically ( Fig. 5). The HPLC fingerprint of Baizhi consisted mainly of 22 peaks, and the attribution of 10 peaks in the profile was confirmed by comparison of the retention time, UV spectrum of reference substances profile, spiking some of the chemical reference substances, as well as re- ferring to the data published in the literature [21]. The attribution of the ten peaks was: adenosine (1), xanthotoxol (6), xanthotoxin (10), oxypeucedanin hydrate (12), isopimpinellin (14), bergapten (16), oxypeucedanin (18), imporatorin (19), cnidilin (21), and isoimporatorin (22). The peaks 10, 12, 16, 17, 18, 19, 21, and 22 dominated the profile of the sun-dried authenticated Baizhi; among them, peak 19 (imporatorin) was the uppermost obvious one ( Fig. 5). The HPLC profile of sulfur-fumigated Baizhi demonstrated that all the major furocoumarins disappeared significantly; peak 19 (imporatorin) had about a 60\% loss; peak 17 and peak 18 (oxypeucedanin) almost disappeared. However, the absorption abundance of peaks 3-11 ( $T R=18-44 \mathrm{~min}$ ) increased to some extent ( Fig. 5). Similarity calculation and principle component analysis (PCA) showed sulfur-fumigated Bai-Zhi has a correlation coefficient lower than 0.85 ( $\bullet$ Fig. 6 ), and two groups are clearly divided between the sun-dried and sulfur-fumigated BaiZhi ( Fig. 7). This confirms that sulfur fumigation significantly damages the inherent quality of Baizhi. The destructive effect of sulfur fumigation to the bioactive constituents has also been confirmed by an imitating test. The literature on modern pharmacological and clinical studies revealed that the furocoumarins in the roots of Baizhi possess various biological activities such as antiinflammation, antitumor, antimicrobial, central analgesic, hepatoprotective, and others. Significant loss of the furocoumarins implies a serious decline in its bioactivity. A published paper re- 


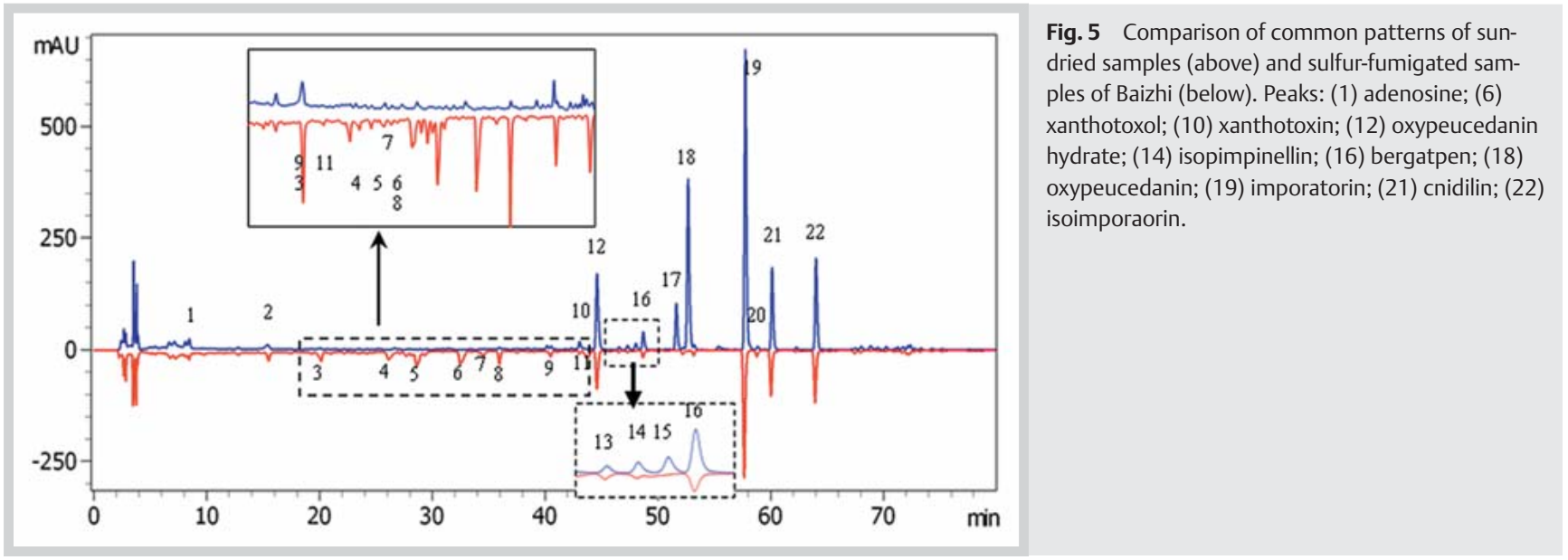

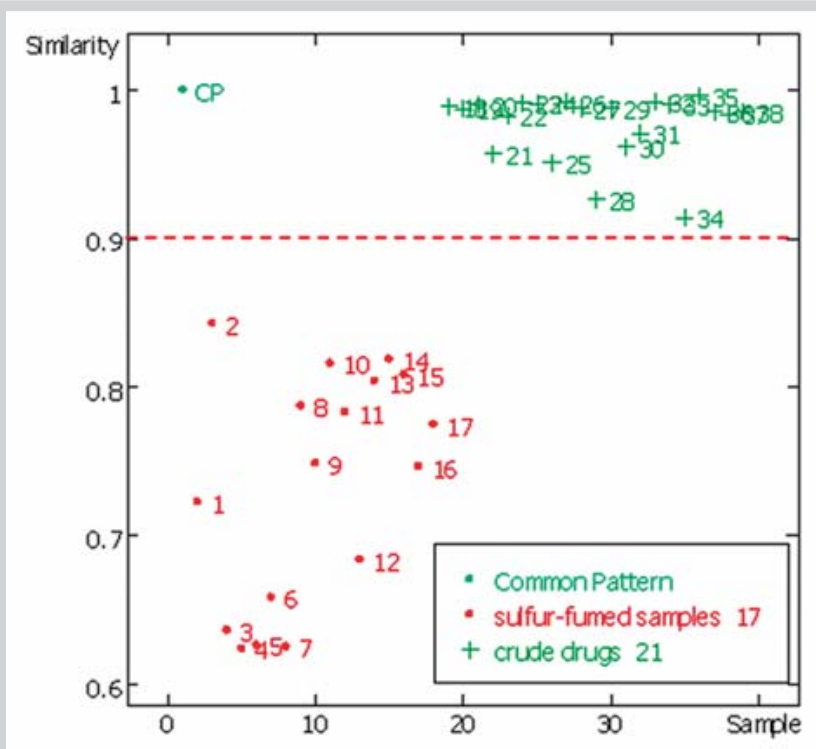

Fig. 6 Similarity of the HPLC fingerprints of Angelicae Formosanae Radix (Bai Zhi).

ported that the analgesic effect of sulfur-fumigated Baizhi decreased drastically compared to the sun-dried Baizhi. The sulfurfumigation process, which results in significant losses of the main active constituents, may consequently be an unacceptable approach for processing CMM in the future.

Chaihu (Bupleuri Radix), roots of Bupleurum chinense and B. scorzonerifolium, is an important Chinese herb in the Chinese pharmacopoeia. Some other species such as the roots of B. falcatum, B. bicaule, and B. marginatum var. stenophyllum which are similar to Chaihu can also be occasionally found in local raw herb markets. The quality of 33 lots of authenticated Chaihu samples vs. 31 lots of commercial samples was evaluated by both high-performance liquid chromatography-evaporative light scattering detector (HPLC-ELSD) and high-performance thin-layer chromatography (HPTLC) analyses of its principal bioactive components (saikosaponins). The pretreated data acquired from both HPLC fingerprints and HPTLC fluorescent images were processed by chemometrics for similarity and pattern recognition, including artificial neural networks (ANNs), $k$-nearest neighbor ( $k$-NN),

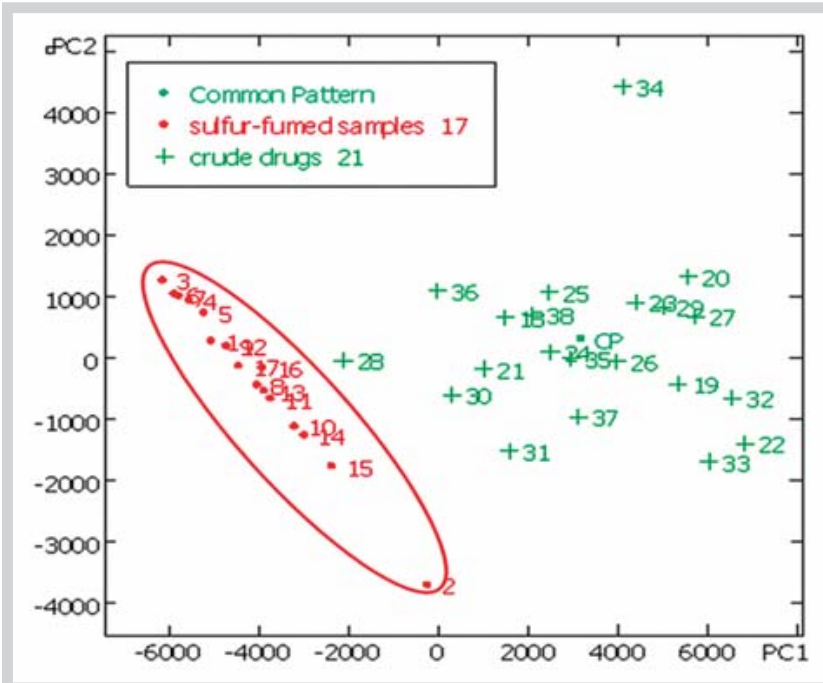

Fig. 7 PCA analysis of HPLC fingerprints of Angelicae Formosanae Radix (Bai Zhi).

and an expert's panel. It was apparent that $k$-NN classifier exhibited good performance with sufficient flexibility for processing HPTLC fingerprint images which were otherwise not easily dealt with by other algorithms due to the shift of $R \mathrm{f}$ values and varying hue/saturation of the band colors between different TLC plates. These two chromatographic fingerprint methods can be considered as a complementary measure of quality control. The roots of Chaihu from different species of the genus Bupleurum could be readily distinguished from each other so that commercial samples can easily be classified. Chaihu collected from several major CMM distribution centers was found to belong to B. chinense with great variation in the content of its major saikosaponins [22]. In summary, chromatographic fingerprinting and simultaneous determination of multiple components as shown above can be one of the important methods for the comprehensive quality control of herbal crude drugs in TCM. 


\section{Conclusion}

\section{$\nabla$}

Facing the downhill slide of quality of herbal crude drugs in the present market, the pragmatic comprehensive chromatographic fingerprint analysis can determine the detectable ingredients composition and concentration distribution under quantifiable operation condition, and therefore provides real-time quality information. It consists of a group of detectable chemical compounds - the peak/band numbers, peak/band position (retention time in HPLC/GC, or migration distance/Rf value in HPTLC) and peak-to-peak ratios (as well as the band color in HPTLC) - in a whole as the unique common pattern of the given species for identification and rapid estimation of the ingredients concentration distribution. A rational acceptable judgment can be made accordingly. It may be a "gray" entity at the primary stage with little known background, but for identification $\mathrm{CF}$ is a good additional methodology and directly beneficial for quality assessment of the crude CMM and proprietary preparations containing CMM. An organized database of $\mathrm{CF}$ generated from authenticated CMM with continuously updated research and market data will increase the knowledge and reduce its "gray scale", increase the transparency gradually and thereby strengthen its quality assessment potency.

\section{References}

1 Oka H, Yamamoto S, Kuroki T, Harihara S, Marumo T, Kim SR, Monna T, Kobayashi K, Tango T. Prospective study of chemoprevention of hepatocellular carcinoma with Sho-saiko-to (TJ-9). Cancer 1995; 76: 743-749

2 Sheehan MP, Atherton D. A controlled trial of traditional Chinese medicinal plants in widespread non-exudative atopic eczema. Br J Dermatol 1992; 126: 179-184

3 Sheehan MP, Rustin MH, Atherton DJ, Buckley C, Harris DW, BrostoffJ, Ostlere L, Dawson A. Efficacy of traditional Chinese herbal therapy in adult atopic dermatitis. Lancet 1992; 340: 13-17

4 Chaudhury RR. Herbal medicine for human health. New Delhi, India: WHO Regional Publication; 1992: 26-27

5 Chan $K$. Chinese medicinal materials and their interface with Western medical concepts. J Ethnopharmacol 2005; 96: 1-18

6 Liang YZ, Xie PS, Chan K. Quality control of herbal medicines. J Chromatogr B 2004; 812: 53-70

7 Ganzera $M$. Recent advancements and applications in the analysis of traditional Chinese medicines. Planta Med 2009; 75: 776-783
8 Liu S, Yi LZ, Liang YZ. Traditional Chinese medicine and separation science. J Sep Sci 2008; 31: 2113-2137

9 Li PK, Guo BL, Huang WH. Studies on fingerprinting and identification of main species of Herba Epimedii. Chin J Chin Mater Med 2008; 33 : 1662-1668

10 Guo BL, Pei LK, Xiao PG. Further research on taxonomic significance of flavonoids in Epimedium (Berberidaceae). J Sys Evol 2008; 46: 874-885

11 Huang CY, Zhao LH, Mei LH. Chin. Studies on the HPLC fingerprint of Epimedium brevicornum crude drug. J Nat Med 2003; 1: 146-148

12 Wang LX, Wang CZ, Geng XD. Studies on the RPLC fingerprints and quality evaluation of Epimedium L. crude drugs. Acta Chim Sin 2006; 64: 551-555

13 Stearn WT. Epimedium and Vancouveria (Berberidaceae). J Linn Soc 1938; 51: 409-535

14 Stearn WT. The genus Epimedium and other herbaceous Berberidaceae including the genus Podophyllium. Oregon: Timber Press; 2002: 35-36

15 Xie PS, Yan YZ, Guo BL, Lam CWK, Chui SH, Yu QX. Chemical patternaided classification to simplify the intricacy of morphological taxonomy of Epimedium species using chromatographic fingerprinting. J Pharm Biomed Anal 2010; 52: 452-460

16 Xu XN, Jiang JH, Liang YZ, Yi LZ, Cheng JL. Chemical fingerprint analysis for quality control of Fructus Aurantii Immaturus based on HPLC-DAD combined with chemometric methods. Anal Meth, advance online publication 2010; doi: 10.1039/C0AY00455C

17 Zhao YY, Zhang YM, Lin RC, Sun WJ. An expeditious HPLC method to distinguish Aconitum kusnezoffii from related species. Fitoterapia 2009; 80: 333-338

18 Yi LZ, Yuan DL, Liang YZ, Xie PS, Zhao Y. Fingerprinting alterations of secondary metabolites of tangerine peels during growth by HPLC-DAD and chemometric methods. Anal Chim Acta 2009; 649: 43-51

19 Mouly P, Gaydou EM, Auffracy A. Simultaneous separation of flavanone glycosides and polymethoxylated flavones in citrus juices using liquid chromatography. J Chromatogr A 1998; 800: 171-179

20 Wang XH, Xie PS, Lam CWK, Yan YZ, Yu QX. Study of the destructive effect to inherent quality of Angelicae dahuricae radix (Baizhi) by sulfurfumigated process using chromatographic fingerprinting analysis. J Pharm Biomed Anal 2009; 49: 1221-1225

21 Kang J, Zhou L, Sun JH, Han J, Guo DA. Chromatographic fingerprint analysis and characterization of furocoumarins in the roots of Angelica dahurica by HPLC/DAD/ESI-MSn technique. J Pharm Biomed Anal 2008; 47: 778-785

22 Tian RT, Xie PS, Liu HP. Evaluation of traditional Chinese herbal medicine - Chaihu (Bupleuri Radix) by both high performance liquid chromatographic and high performance thin-layer chromatographic fingerprint and chemometric analysis. J Chromatogr A 2009; 1216: 2150-2155 